\title{
Healthcare Utilization and Comorbidity in Chronic Lymphocytic Leukemia
}

\author{
Emelie C Rotbain (DD ${ }^{1-5}$ \\ Klaus Rostgaard $\mathbb{D}^{3,5}$ \\ Michael A Andersen ${ }^{2,3}$ \\ Caspar Da Cunha-Bang ${ }^{2}$ \\ Carsten U Niemann $\mathbb{D D}^{2}$ \\ Henrik Frederiksen (ID ${ }^{1,4,6}$ \\ Henrik Hjalgrim ${ }^{2,3,5,7}$ \\ 'Department of Hematology, Odense \\ University Hospital, Odense, Denmark; \\ ${ }^{2}$ Department of Hematology, Rigshospitalet, \\ Denmark; ${ }^{3}$ Department of Epidemiology \\ Research, Statens Serum Institut, \\ Copenhagen, Denmark; ${ }^{4}$ Department of \\ Clinical Research, University of Southern \\ Denmark, Odense, Denmark; ${ }^{5}$ Danish \\ Cancer Society Research Center, \\ Hematology Research Group, Copenhagen, \\ Denmark; ${ }^{6}$ Academy of Geriatric Cancer \\ Research (AGECARE), Odense University \\ Hospital, Odense, Denmark; ${ }^{7}$ Department \\ of Clinical Medicine, Copenhagen University, \\ Copenhagen, Denmark
}

Correspondence: Emelie C Rotbain Department of Hematology, Odense University Hospital, Kløvervænget 6,

Odense, DK-5000, Denmark

Tel +45 53690201

Emailerot0006@regionh.dk
Purpose: Age-related comorbidity is highly prevalent in chronic lymphocytic leukemia (CLL). The purpose of this study was to provide information on current patterns of healthcare utilization in CLL.

Patients and Methods: We used data from Danish nation-wide registers to study healthcare utilization the year before and the year after CLL diagnosis and in relation to first-line treatment. Patients diagnosed with CLL between 1997 and 2018 were included and stratified on number of comorbidities, presence of specific comorbidities, and fitness status, respectively. Healthcare utilization was studied in terms of hospital admissions, in-hospital bed days, out-patient visits, emergency room visits, and prescription drugs. Odds ratios with 95\% confidence intervals were calculated using multivariable logistic regression analyses adjusting for age, sex, and calendar year.

Results: The study comprised 9170 patients with CLL with a median age of 71 years, of whom $35 \%$ had $\geq 1$ comorbidity. Healthcare utilization increased markedly upon CLL diagnosis both in patients with and without comorbidities. During the year after CLL diagnosis, 39\% were hospitalized, $16 \%$ visited an emergency room, $88 \%$ visited an outpatient clinic, and $93 \%$ received prescription drugs. Both individual comorbidities and the total number of comorbidities were associated with increased healthcare utilization of all types, except for contacts to hematological departments.

Conclusion: Our results suggest that CLL diagnosis may unveil incipient diseases and aggravate comorbidities and thereby have considerably wider health implications than those directly related to CLL. These findings may be used by clinicians and decisions makers to guide planning of multidisciplinary care for cancer patients.

Keywords: chronic lymphocytic leukemia, hematology, epidemiology, real-world data

\section{Introduction}

Over the last two decades, advances in chronic lymphocytic leukemia (CLL) treatment have markedly improved patient survival. ${ }^{1,2}$ Diagnosis of CLL typically marks the beginning of a lifelong follow-up program for management and treatment of the disease and of related complications at hematological department. As the median age at CLL diagnosis is 72 years, many newly diagnosed CLL patients have one or more chronic comorbidities. $^{3-7}$ When complicated by CLL, the responsibility for the management and treatment of other chronic conditions often become more difficult to determine. ${ }^{8}$ Thus, lack of coordination between healthcare professionals is common in cancer care, and may aggravate the course of comorbid conditions. ${ }^{9}$

With a continuously aging cancer population and increasing prevalence of multimorbidity (ie, $\geq 2$ chronic conditions), the planning and implementing of 
coordinated, interdisciplinary efforts become increasingly important. As recently highlighted by Rotenstein et al, there is a lack of information on existing healthcare utilization (HCU) patterns among persons with cancer and coexisting conditions, leaving the scope of the required interventions largely uncharted. ${ }^{10}$

The purpose of this study was to inform the discussion of healthcare planning for patients with CLL and comorbidities. We assessed HCU for patients based on comorbidity or frailty status in the periods immediately before and after diagnosis and upon treatment in a nationwide cohort study with complete information on hospital contacts and use of prescription medications.

\section{Methods}

\section{Data Sources and Study Population}

In Denmark, all citizens have access to free healthcare, and contacts to health services are monitored through national administrative health registers. Each individual has a unique personal identification number, which allows for linkage of data across national registers on personlevel. ${ }^{11}$ The Danish National CLL Register is a clinical quality register containing data on all patients diagnosed with CLL since 2008. Entry of data on CLL patients is mandatory for treating physicians. ${ }^{12}$ For this study, we combined records from the Danish National CLL Register with data from several national administrative health registers.

The study population comprised all patients registered with CLL in the CLL register 2008-2017 or the Danish Cancer Register (DCR) 1997-2018. ${ }^{12,13}$ Patients <30 years of age at CLL diagnosis were excluded to avoid inclusion of other lymphoma sub-types misclassified as CLL. Information on death and migration was available from the Danish Civil Registration System. ${ }^{14}$

The Danish National Patient Register (DNPR) contains information for all admissions since 1978, and all hospitalbased out-patient and emergency room visits since 1995 and was used to attain information on hospital contacts. ${ }^{15}$ The Danish National Prescription Registry was used to obtain information on all drugs prescribed. ${ }^{16}$ We used the 4th level (5 digits) of the Anatomical Therapeutic Chemical Classification System to count the number of different drugs prescribed. The following types of HCU were studied: in-hospital bed days, emergency room visits, hospitalizations, out-patient clinic visits, and prescription drugs. For patients residing in the Eastern Region of
Denmark, hospitalizations and out-patient clinic visits were disaggregated into hematological or nonhematological departments, respectively.

Comorbidity was assessed 30 days after CLL diagnosis using ICD-8 and ICD-10 discharge codes from the DNPR, with a look-back period of up to 20 years. Inclusion of comorbid conditions were based on a modified version of the Charlson Comorbidity Index (CCI) previously used by the investigators. ${ }^{7,17,18}$ Comorbidity status was defined as number of comorbid conditions $(0,1$ or $\geq 2)$ or presence of the four most common comorbidities individually: diabetes, chronic pulmonary disease, congestive heart failure or myocardial infarction (MI), and cerebrovascular disease.

Data on CLL first-line treatment was available for patients in the CLL register. ${ }^{12}$ Treated patients were categorized as fit if they received fludarabine, cyclophosphamide, and rituximab (FCR) or bendamustine and rituximab (BR) and as frail if they received chlorambucil as monotherapy $(\mathrm{Clb})$ or in combination with anti-CD20 antibodies. In Denmark, few treatment-naïve patients receive targeted agents, as this is only recommended for patients with TP53-aberrations in Danish CLL guidelines. Therefore, targeted agents were not studied, as we expected treatment-naïve patients to mostly receive chemotherapy-based treatment.

\section{Statistical Analysis}

Descriptive statistics containing numbers and percentages for categorical and ordinal variables were calculated. Median and interquartile range was calculated for continuous, non-normally distributed variables.

Univariable and multivariable logistic analyses were performed to calculate odds ratios (OR) for $\mathrm{HCU}$ for patients based on number of comorbidities and presence of specific comorbidities, during the year after CLL diagnosis. ORs were also calculated for HCU based on fitness status before, during, and after CLL treatment. All periods related to treatment were 6 months each. Patients who received treatment within 6 months after CLL diagnosis were not included in the analysis of HCU before treatment. Furthermore, we calculated ORs for HCU the year after CLL diagnosis compared with the year before, stratifying by number of comorbidities. The last month before and the first month after CLL diagnosis was not included, as to exclude the period of medical work up for CLL.

Multivariable logistic regression analyses included adjustment for age, sex, and calendar year. For comparing 
HCU before and after CLL diagnosis, sensitivity analyses were also performed excluding patients who started CLL treatment within one year after diagnosis. Results are presented using ORs with 95\% confidence intervals (CI).

For modalities of healthcare that were utilized by less than $50 \%$ of patients during the first year after CLL diagnosis, the outcome was defined as having $\geq 1$ utilization during the period of interest. For modalities of healthcare that were utilized by more than $50 \%$ of patients during the first year after CLL diagnosis the median number of usages during the first year after CLL diagnosis was used as a cut-off for the outcome. For in-hospital bed days, the median number was 0 and the 75 th percentile was used as the cut-off instead. This resulted in the following outcomes: $\geq 5$ in-hospital bed days, $\geq 1$ emergency room visit, $\geq 1$ hospitalization, $\geq 5$ out-patient clinic visits, and $\geq 6$ prescription drugs during the period of interest. In analyses of $\mathrm{HCU}$ before, during, and after treatment, where study periods were only 6 months instead of 1 year, $\geq 3$ in-hospital bed days was used as cut-off instead of $\geq 5$ in-hospital bed days.

Data analysis was performed using SAS software, version 9.4 (SAS Institute) and R software version 3.5.2 on servers hosted by Sundhedsdatastyrelsen (Danish Health Data Authority).

\section{Ethics}

The study was approved by the Danish Health and Medicine Authorities (jr. no 3-3013-1141/1) and the Danish Data Protection Agency (jr.no RH-2015-9603856).

\section{Results}

\section{Baseline Characteristics}

Baseline characteristics for 9170 CLL patients included in the study are presented in Table 1 and detailed data for this cohort has been published previously. ${ }^{7}$ The population consisted predominantly of males $(60 \%)$ and the median age at CLL diagnosis was 71 years. Comorbidity was present in $35 \%$ of patients; $23 \%$ had 1 comorbidity and $12 \%$ had $\geq 2$ comorbidities. The prevalence of diabetes, chronic pulmonary disease, congestive heart failure or MI, and cerebrovascular disease was $8 \%, 8 \%, 10 \%$, and $9 \%$, respectively. Among patients treated with the most common treatment regimens, 59\% were considered fit and $41 \%$ frail. During the year after CLL diagnosis, 39\% had been hospitalized, 16\% had visited an emergency room, and 88\% had visited a hospital out-patient clinic. Moreover, 93\% had received prescription medications with a median number of 6 drugs during the year after CLL diagnosis.

\section{HCU After CLL Diagnosis Compared to Prior}

In Table 2, HCU the year after CLL diagnosis is compared with the year prior to CLL diagnosis in the same patients sub-grouped on number of comorbidities. All types of HCU statistically significantly increased after CLL diagnosis regardless of number of comorbidities (OR range: 1.25-8.78), except for emergency room visits and outpatient visits at non-hematological departments which did not increase for patients with $\geq 2$ comorbidities. HCU correlated with number of comorbidities both before and after CLL diagnosis, while patients without comorbidities experienced the greatest relative increase in HCU after CLL diagnosis. Similar results were found when restricting the analysis to patients who did not receive treatment the first years after diagnosis (Supplemental Table 1).

Figure 1 displays cumulative percentage frequency distributions of bed days before and after CLL diagnosis for patients stratified on number of comorbidities. Regardless of number of comorbidities, a small percentage of patients accounted for the vast majority of bed days. In patients without comorbidities at CLL diagnosis, $16.6 \%$ accounted for $90 \%$ of all bed days after CLL diagnosis (Figure 1A). Similar skewed distributions were observed for patients with either 1 or $\geq 2$ comorbidities at CLL diagnosis, respectively (Figure 1B and C).

\section{Number of Comorbidities and $\mathrm{HCU}$}

Table 3 contains ORs for HCU the year after CLL diagnosis comparing patients with 0,1 or $\geq 2$ comorbidities. For 4137 patients residing in the Eastern Region of Denmark, use of hematological and non-hematological departments, were also studied. All types of HCU statistically significantly increased with the number of comorbidities (OR range: 1.28-7.59), except for contacts to hematological departments.

\section{Specific Comorbidities and HCU}

A comparison of $\mathrm{HCU}$ in CLL patients with diabetes, chronic pulmonary disease, congestive heart failure or $\mathrm{MI}$, or cerebrovascular disease, respectively, to CLL patients without the same condition is displayed in Figure 2 and Supplemental Table 2. All comorbidities were individually associated with bed days (OR range: 1.21-1.28), except for cerebrovascular disease, while 
Table I Baseline Characteristics for Chronic Lymphocytic Leukemia Patients at Time of Diagnosis and Healthcare Utilization Calculated for the Year After Chronic Lymphocytic Leukemia Diagnosis

\begin{tabular}{|c|c|}
\hline & $\begin{array}{l}\text { All Patients } \\
\mathbf{N}=9170\end{array}$ \\
\hline \multicolumn{2}{|l|}{ Sex } \\
\hline Female & $3687(40)$ \\
\hline Male & $5483(60)$ \\
\hline \multicolumn{2}{|l|}{ Age at diagnosis (years) } \\
\hline Median [IQR] & $71[63,79]$ \\
\hline$<60$ & $1629(18)$ \\
\hline $60-69$ & $2600(28)$ \\
\hline $70-79$ & $3014(33)$ \\
\hline$>80$ & $1927(21)$ \\
\hline \multicolumn{2}{|l|}{ Calendar year } \\
\hline $1997-2002$ & $1954(21)$ \\
\hline $2003-2008$ & $2273(25)$ \\
\hline $2009-2013$ & $2277(25)$ \\
\hline $2014-2018$ & $2666(29)$ \\
\hline \multicolumn{2}{|l|}{ Comorbidities } \\
\hline 0 & $5916(65)$ \\
\hline 1 & $2133(23)$ \\
\hline$\geq 2$ & $1121(12)$ \\
\hline Diabetes & $751(8)$ \\
\hline Chronic pulmonary disease & $722(8)$ \\
\hline Congestive heart failure or myocardial infarction & $914(10)$ \\
\hline Cerebrovascular disease & $785(9)$ \\
\hline \multicolumn{2}{|l|}{ Fitness status* } \\
\hline Fit & $597(59)$ \\
\hline Frail & $4 I 7(4 I)$ \\
\hline \multicolumn{2}{|l|}{ Hospitalization } \\
\hline Yes & 3564 (39) \\
\hline Median number of bed days [QI, Q3] & $0[0,5]$ \\
\hline \multicolumn{2}{|l|}{ Emergency room } \\
\hline Yes & $1428(16)$ \\
\hline \multicolumn{2}{|l|}{ Out-patient clinic } \\
\hline Yes & $8051(88)$ \\
\hline Median number of visits [QI, Q3] & $5[2,10]$ \\
\hline \multicolumn{2}{|l|}{ Prescription drugs } \\
\hline Yes & $8529(93)$ \\
\hline Median number of different drugs [QI, Q3] & $6[3,9]$ \\
\hline
\end{tabular}

Notes: *Based on a subset of patients from a geographically defined area. Abbreviation: IQR, interquartile range.

diabetes was the only comorbidity not associated with hospitalization (OR range for other comorbidities: 1.191.29). All conditions were associated with prescription drugs usage (OR range: 1.45-3.12). Chronic pulmonary disease (OR: 1.23 [1.01; 1.51]) and congestive heart failure or MI (OR: 1.22 [1.01; 1.48]) were both associated with emergency room visits, whereas diabetes was associated with out-patient clinic visits (OR: 1.37 [1.16; 1.61]).

\section{Frailty and HCU Upon Treatment}

HCU before, during, and after first-line treatment for patients who were considered frail or fit, respectively, based on treatment regimens received are presented in Table 4. Frailty was associated with prescription drugs usage during all periods (OR range: 1.68-2.27) and with out-patient clinic visits after treatment (OR: 1.59 [1.10; 2.29]). No statistically significant association was observed between frailty and bed days, emergency room visits, or hospitalizations.

\section{Discussion}

In modern cancer care, inadequate coordination with other medical specialties resulting in exacerbation of comorbid conditions is common. ${ }^{9}$ With a median age at diagnosis of 72 years and continuously improving survival, patients diagnosed with CLL may be particularly at risk hereof because of frequent age-related comorbidities.

Our results provide evidence that CLL diagnosis is accompanied by an aggravated course of comorbidities as measured by selected HCU modalities. Thus, in line with previous Danish cancer studies, all types of investigated HCU increased the first year after CLL diagnosis compared with the year before CLL diagnosis in our study. ${ }^{19}$ For outpatient visits at hematological departments, this increase is expected, as patients with CLL are followed at hematological departments with regular visits, typically ranging from 1 to 4 times per year in the watch and wait setting. Due to the immune dysfunction observed in CLL, also in patients not requiring treatment, the threshold for admitting patients upon suspicion of infection may be lower in CLL patients, leading to high numbers of hospitalization. ${ }^{20,21}$ While this approach may lead to overdiagnosing of infections in CLL patients compared with their counterparts with similar comorbidities and no CLL, we believe it is a necessary precaution considering the high rate of death due to infections in CLL. ${ }^{1,22}$ Still, the high number of planned and unplanned hospital visits and admission by patients with CLL places a high burden on the healthcare system, both at non-hematological and hematological departments. Future studies are required to uncover interventions that may reduce unplanned hospitalizations and emergency room visits in patients with CLL. ${ }^{23}$

Overall, HCU increased with number of comorbidities both before and after CLL diagnosis. With $31-38 \%$ of patients having $\geq 5$ in-hospital bed days during the year after CLL diagnosis and $68-84 \%$ receiving $\geq 6$ prescriptive drugs, the burden of HCU is extremely high in comorbid 
Table 2 Healthcare Utilization During the First Year Post Chronic Lymphocytic Leukemia Diagnosis Compared with the Year Prior to Chronic Lymphocytic Leukemia Diagnosis for the Same Patients Stratified by the Number of Comorbidities

\begin{tabular}{|c|c|c|c|c|c|c|c|c|c|}
\hline & \multicolumn{3}{|c|}{ No Comorbidity } & \multicolumn{3}{|c|}{ I Comorbidity } & \multicolumn{3}{|c|}{$\geq 2$ Comorbidity } \\
\hline & \multicolumn{2}{|c|}{ Percent Utilizing } & \multirow{2}{*}{$\begin{array}{l}\text { OR }(95 \% \mathrm{Cl}) \\
\text { Post vs Prior }\end{array}$} & \multicolumn{2}{|c|}{ Percent Utilizing } & \multirow{2}{*}{$\begin{array}{l}\text { OR }(95 \% \mathrm{Cl}) \\
\text { Post vs Prior }\end{array}$} & \multicolumn{2}{|c|}{ Percent Utilizing } & \multirow{2}{*}{$\begin{array}{l}\text { OR }(95 \% \mathrm{CI}) \\
\text { Post vs Prior }\end{array}$} \\
\hline & Prior & Post & & Prior & Post & & Prior & Post & \\
\hline$\geq 5$ in-hospital bed days & 6 & 21 & $4.43(3.91-5.03)$ & 16 & 31 & $2.29(1.97-2.65)$ & 29 & 38 & $1.5 \mathrm{I}(\mathrm{I} .27-1.8 \mathrm{I})$ \\
\hline Emergency room & 9 & 13 & $1.55(1.37-1.74)$ & 14 & 18 & $1.34(1.14-1.58)$ & 22 & 25 & $1.14(0.94-1.38)$ \\
\hline Hospitalizations & 13 & 34 & $3.48(3.17-3.82)$ & 26 & 45 & $2.29(2.02-2.6 \mathrm{I})$ & 40 & 55 & $1.88(1.59-2.22)$ \\
\hline Hospitalizations, hem department* & $<1$ & 12 & $41.7(21.5-81.1)$ & $<1$ & $\mathrm{II}$ & $39.2(12.4-124)$ & $<1$ & 12 & $36.3(8.84-149)$ \\
\hline Hospitalizations, non-hem department* & 15 & 27 & $2.13(1.85-2.44)$ & 28 & 37 & $1.57(1.29-1.91)$ & 43 & 51 & $1.40(1.10-1.79)$ \\
\hline$\geq 5$ out-patient clinic visits & 10 & 50 & $8.78(7.96-9.70)$ & 20 & 54 & $4.57(3.99-5.24)$ & 32 & 59 & $3.11(2.62-3.70)$ \\
\hline Hem out-patient clinic* & 2 & 69 & $98.5(75.3-129)$ & 3 & 66 & $66.2(44.1-99.3)$ & 4 & 63 & $40.1(25.0-64.2)$ \\
\hline Non-hem out-patient clinic* & 36 & 57 & $2.27(2.04-2.54)$ & 55 & 68 & $1.77(1.47-2.13)$ & 73 & 74 & $1.06(0.81-1.40)$ \\
\hline$\geq 6$ prescription drugs & 27 & 38 & $1.65(1.52-1.78)$ & 58 & 68 & $1.53(1.35-1.74)$ & 81 & 84 & $1.25(1.01-1.56)$ \\
\hline
\end{tabular}

Notes: *Based on a subset of patients from a geographically defined area.

Abbreviation: Hem, hematological.

patients with CLL. Our findings are in line with previous findings in lymphomas, demonstrating an association between multimorbidity and increased HCU. ${ }^{24}$

This increase in HCU the first year after CLL diagnosis compared with the year before was seen irrespective of number of comorbidities but was relatively largest for patients without known comorbid conditions at time of CLL diagnosis. Thus, of non-comorbid patients with CLL, no less than one-third were hospitalized during the first year after CLL diagnosis, and half of the patients had $\geq 5$ out-patient clinic visits. These findings illustrate that even among patients without comorbidities the total burden of disease associated with diagnosis of CLL is far from negligible. While the increased HCU is likely partly related to intensified scrutiny of patients as a result of a new diagnosis, the considerable surge of healthcare contacts still necessitates coordination of care.

At the same time as our results highlight an increased HCU following CLL diagnosis they also indicate that the degree of intensification in HCU is not evenly distributed among patients. Thus, both before and after CLL diagnosis a small proportion of patients accounted for the vast majority of in-hospital bed days. This skewed distribution was observed irrespective of number of comorbidities at time of CLL diagnosis, suggesting that subsets of frail patients with CLL exist who might benefit from close surveillance in conjunction to cancer diagnosis. Further studies are essential to identify which patients without previously known comorbidities may have an increased use of healthcare services and need for more closely coordination care after CLL diagnosis.
Previous findings show that HCU has increased in CLL over recent years, further supporting the need for formalized collaborations in multidisciplinary teams to coordinate personalized care for comorbid patients upon cancer diagnosis. $^{25}$ Several models for this work are currently being developed in oncological cancer care, while evidence-based methods and general uptake are lacking in the hematological setting. ${ }^{9,26,27}$

With a median number of 6 prescription drugs, polypharmacy was highly frequent in our population, even among CLL patients without comorbidities. These findings are consistent with previous studies of similarly aged populations with different types and stages of cancer, where the median number of prescribed drugs were 5-7, with one study even reporting a median between 9 and 11 across sub-groups. ${ }^{28-32}$ Previous findings also show that only a minority of cancer patients receiving the most commonly taken drugs: anti-hypertensives and lipidlowering agents, have a history of cardiovascular disease (including MI, angina, cerebrovascular accident, and transient ischemic attack), possibly explaining the high frequency of polypharmacy in non-comorbid patients in our study. ${ }^{28}$ The importance of management of polypharmacy is highlighted by the association of polypharmacy with increased risk of interaction or contraindication of prescribed drugs. ${ }^{29}$ Coordinated multidisciplinary care including pharmaceutical teams and polypharmacy clinics may help deprescribing inappropriate medications in cancer patients. $^{33}$

As diabetes is often treated with multiple anti-diabetic drugs and multifactorial treatment, it is unsurprising that 
A

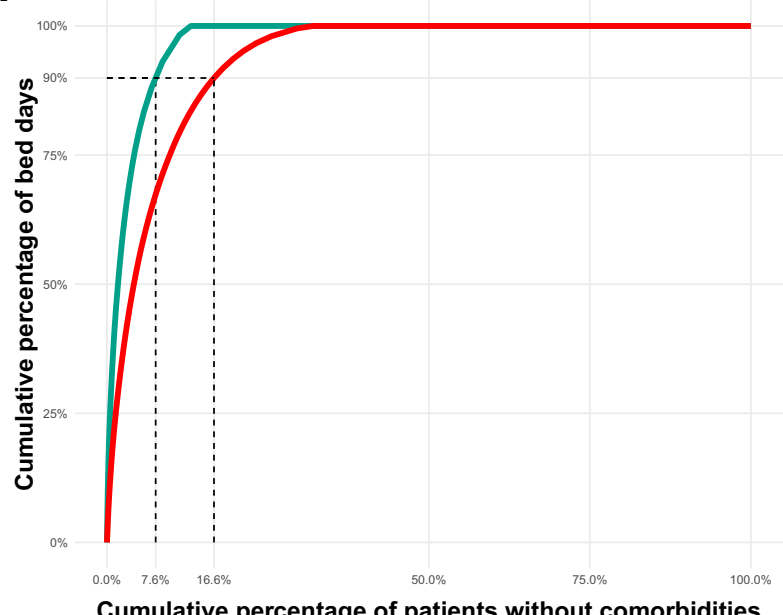

B

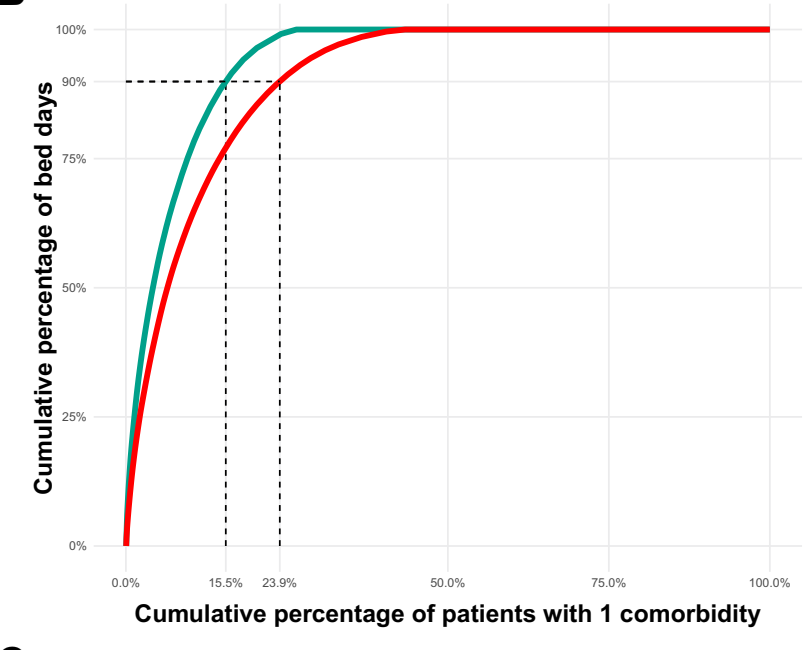

C

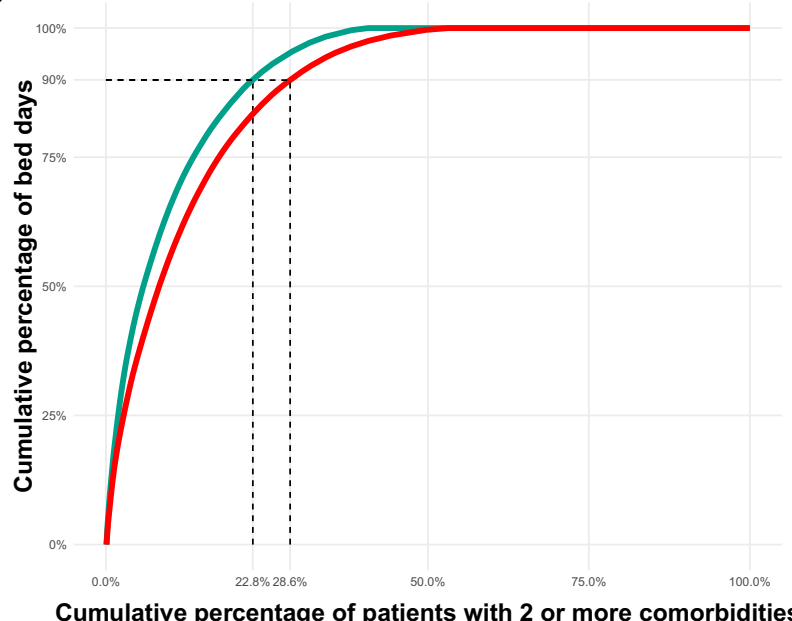

Time point - After CLL diagnosis - Before CLL diagnosis

Figure I Inverted Lorenz curves for in-hospital bed days. In-hospital bed days for patients with CLL without comorbidities (A), with I comorbidity (B), or with 2 or more comorbidities (C), the year before and the year after CLL diagnosis. Inverted Lorenz curves are illustrating cumulative percentage frequency distributions of in-hospital bed days. The $\mathrm{x}$ axis shows percentage of bed days and the $\mathrm{y}$ axis the cumulative percentage of patients that contributed to the bed days. The reference line illustrates the percentage of the patients who accounted for $90 \%$ of the total number of bed days. 


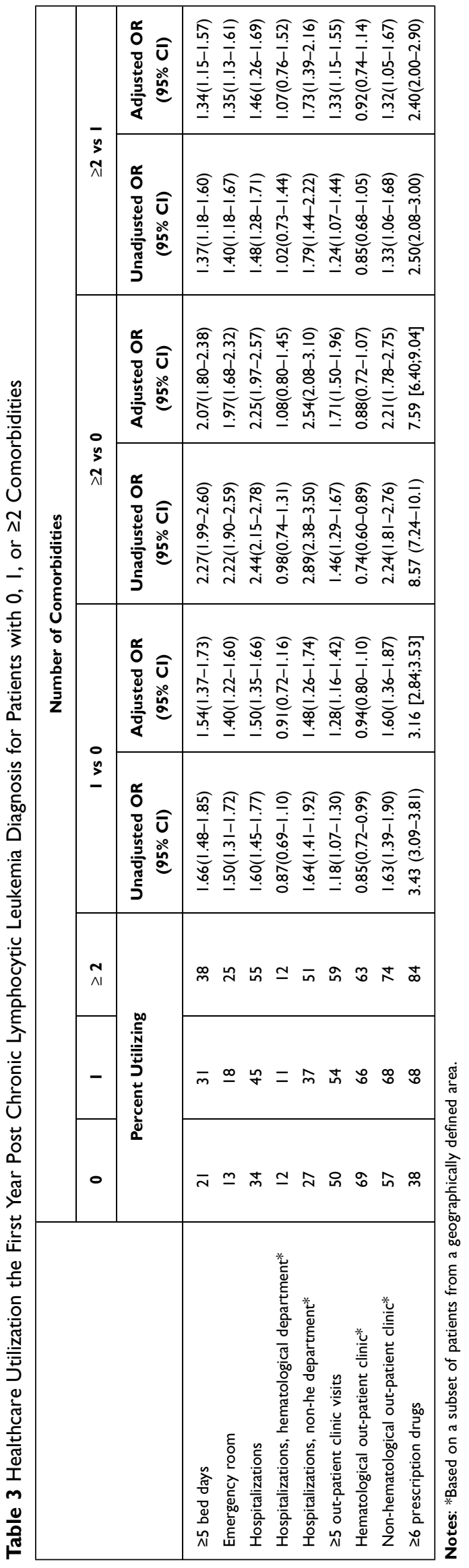

$82 \%$ of CLL patients with diabetes received $\geq 6$ prescriptive drugs. ${ }^{34}$ Previous studies have demonstrated that selfmanagement of diabetes may be poorer during cancer treatment. ${ }^{35}$ It has furthermore been shown among adult patients with acute lymphoblastic leukemia, that poor glycemic control is associated with shorter remission duration, shorter median survival, and increased risk of severe infections during treatment. ${ }^{36}$ However, cancer patients who receive diabetes education are more likely to have multiple HbA1c tests performed per year and fewer hospitalizations. ${ }^{37}$ Closer collaboration between hematologists/oncologists and endocrinologists are required to manage the complicated relationship between cancer and diabetes and in particular for monitoring diabetes during cancer treatment. $^{38}$

In accordance with previous findings, congestive heart failure or MI was associated with emergency room visits and hospitalizations the year after CLL diagnosis. ${ }^{10}$ In the era of targeted agents, optimizing treatment for patients with cardiovascular disease or risk factors is of especially high importance. ${ }^{39-42}$ Simultaneous follow-up in general practice and by oncologists may facilitate care for comorbidities as well as preventative care. ${ }^{43-45}$ Close collaboration with other specialist may be essential to initiate and maintain patients on treatment with BTK-inhibitors such as Ibrutinib in a routine care setting. ${ }^{39,46}$ Further studies are required to identify interventions that may increase safety for patients with cardiovascular risk factors such as older age, diabetes, or high blood pressure upon treatment with BTK-inhibitors.

In CLL, the former definition of "no-go patients" who are only candidates for palliative treatment has largely been rejected in recent years due to less toxic yet efficient therapy options. ${ }^{47}$ This is further supported by our findings, showing that frail patients treated with Clb-based regimes did not have more hospital contacts than fit patients treated with intensive CIT including FCR and BR. We hypothesize that physicians are adept at choosing appropriate intensity of treatment for routine care patients. Treatment strategies are plentiful with new targeted drugs and combination therapies continuously being introduced, and prevalence of comorbidity along with prognostic factors and patients' preferences are of high importance when selecting treatment. ${ }^{48}$ Several aspects of comorbidity and frailty needs to be considered when selecting treatment regimen including kidney function and Cumulative Illness Rating Scale score, as well as specific conditions such as cardiovascular disease or risk factors hereof. Real- 


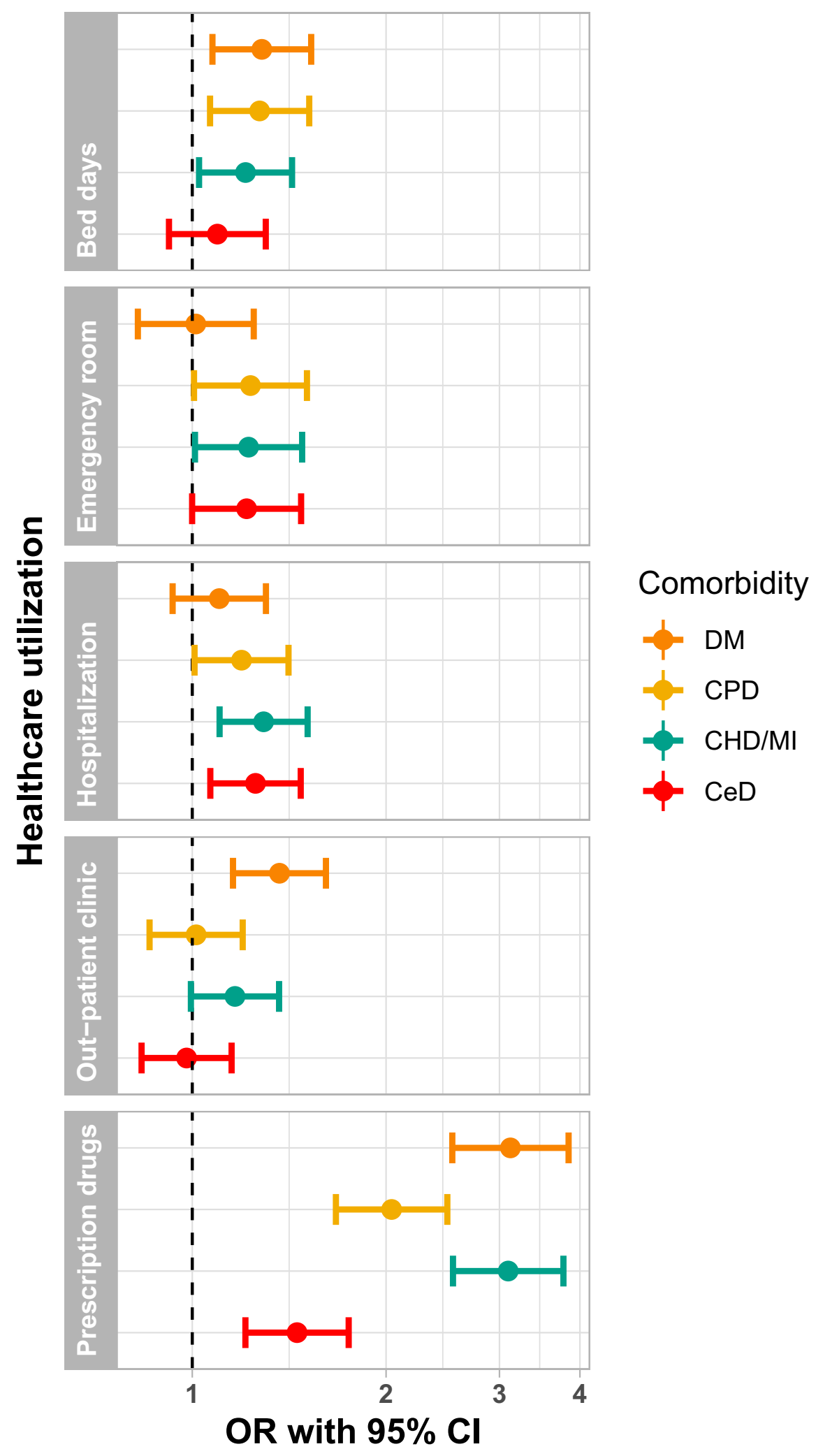

Figure 2 Forest plot of odds ratios for healthcare utilization. Healthcare utilization in terms of $\geq 5$ in hospital bed days, emergency room visits, hospitalizations, $\geq 5$ outpatients clinic visits, and $\geq 6$ prescription drugs during the year after CLL. ORs is for CLL patients with co-existing diabetes (DM), chronic pulmonary disease (CPD), congestive heart failure or MI (CHD/MI), or cerebrovascular disease (CeD) compared with CLL patients without the same specific co-existing condition. ORs are adjusted for age, sex, calendar year, and multimorbidity. ORs are presented on a base-10 logarithmic scale. 


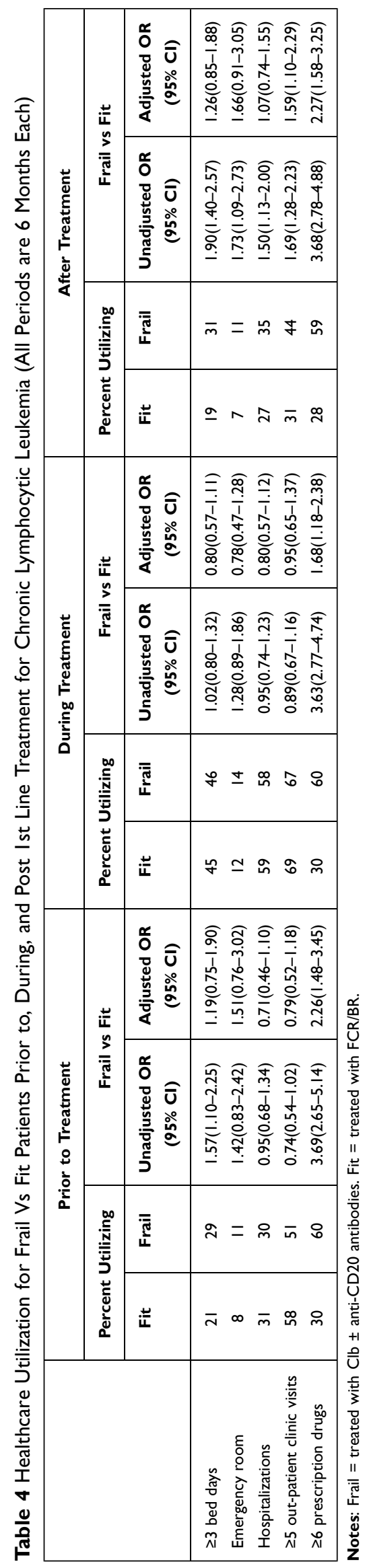

world data from our group and others show that patients receiving Clb-based regimens have a median age of 76-80 years, and it remains to accurately determine these patients' tolerance of targeted treatment and need for supportive care. $^{22,49}$

Data on comorbidities in this study were based on ICD-8 and ICD-10 codes from hospital visits. Thus, comorbidities followed without hospital referral may not have been captured. However, we have included comorbidities coded up until one month after CLL diagnosis to also capture comorbid conditions entered by physicians performing work-up and diagnosing CLL. An important limitation of this study is that comorbidity is more common in older patients who have had a longer time to develop comorbidities. While this association may skew the results, we have adjusted for age in the multivariable analyses. Another limitation is that the course of CLL differs from most cancer types as the majority of CLL patients do not require treatment at the time of diagnosis. Therefore, our finding may not be generalizable to other cancer types.

\section{Conclusion}

Our results demonstrate that $\mathrm{HCU}$ increases markedly upon CLL diagnosis both in patients with and without comorbidities and that $\mathrm{HCU}$ increases with number of comorbidities. An increase in HCU among patients without preexisting comorbidity suggests that CLL diagnosis may unveil incipient diseases and/or aggravate conditions monitored in general practice. Accordingly, CLL diagnosis may have considerably wider health implications than those directly related to the leukemia and its treatment. Our results may be used to guide future personalized multidisciplinary care plans for an increasing number of patients with cancer and comorbidities.

\section{Acknowledgments}

An abstract related to this paper was presented at the 25th Congress of the European Hematology Association Virtual Edition, 2020 as a poster presentation with interim findings. The poster's abstract was published in "Poster Abstracts" in HemaSphere named "Frail patients with CLL can be treated with non-intensive regimens without excess hospitalizations - a nationwide, population-based study": https://library.ehaweb.org/eha/2020/eha25th/ 294632/emelie.curovic.rotbain.frail.patients.with.cll.can. be.treated.with.html 


\section{Funding}

This research was supported in part by Novo Nordisk Foundation grant NNF16OC0019302, AstraZeneca, the Region of Southern Denmark, and the Danish Cancer Society. AstraZeneca was provided with the opportunity to prospectively review the manuscript. The funding sources had no influence on the decision to submit this manuscript, the content of this manuscript, or the interpretation of the data.

\section{Disclosure}

ECR received consultancy fees or travel grants from AbbVie, Janssen, and AstraZeneca. CC-B received consultancy fees and/or travel grants from AbbVie, AstraZeneca, and Gilead. CUN received support from Novo Nordisk Foundation and AstraZeneca within this work and consultancy fees, or travel grants from AbbVie, Gilead, Janssen, Roche, CSL Behring, Acerta, Genmab, Sunesis, Takeda, Octapharma, and AstraZeneca outside this work. HF received support outside this work from Alexion, Gilead, AbbVie, Janssen Pharmaceuticals, and Novartis. HH received support from Neye Fonden outside this work. The authors report no other conflicts of interest in this work.

\section{References}

1. da Cunha-bang C, Simonsen J, Rostgaard K, Geisler C, Hjalgrim H, Niemann CU. Improved survival for patients diagnosed with chronic lymphocytic leukemia in the era of chemo-immunotherapy: a Danish population-based study of 10455 patients. Blood Cancer J. 2016;6: e499. doi:10.1038/bcj.2016.105

2. Siegel RL, Miller KD, Jemal A. Cancer statistics, 2020. CA Cancer J Clin. 2020;70:7-30. doi:10.3322/caac.21590

3. Noone A, Howlader N, Krapcho M, et al. SEER cancer statistics review, 1975-2015, National Cancer Institute. Bethesda, MD; 2018, https://seer.cancer.gov/csr/1975_2015/. Accessed December 15, 2021. based on November 2017 SEER data submission, posted to the SEER web site, April 2018.

4. Strati P, Parikh SA, Chaffee KG, et al. Relationship between co-morbidities at diagnosis, survival and ultimate cause of death in patients with chronic lymphocytic leukaemia (CLL): a prospective cohort study. Br J Haematol. 2017;178:394-402. doi:10.1111/bjh.14785

5. Rigolin GM, Cavallari M, Quaglia FM, et al. In CLL, comorbidities and the complex karyotype are associated with an inferior outcome independently of CLL-IPI. Blood. 2017;129:3495-3498. doi:10.1182/ blood-2017-03-772285

6. Thurmes P, Call T, Slager S, et al. Comorbid conditions and survival in unselected, newly diagnosed patients with chronic lymphocytic leukemia. Leuk Lymphoma. 2008;49:49-56. doi:10.1080/ 10428190701724785

7. Rotbain EC, Niemann CU, Rostgaard K, da Cunha-bang C, Hjalgrim H, Frederiksen H. Mapping comorbidity in chronic lymphocytic leukemia: impact of individual comorbidities on treatment, mortality, and causes of death. Leukemia. 2021;35:2570-2580. doi:10.1038/s41375-41021-01156-x
8. Sada YH, Street RL Jr, Singh H, Shada RE, Naik AD. Primary care and communication in shared cancer care: a qualitative study. $\mathrm{Am}$ J Manag Care. 2011;17:259-265.

9. Lee SJ, Clark MA, Cox JV, Needles BM, Seigel C, Balasubramanian BA. Achieving coordinated care for patients with complex cases of cancer: a multiteam system approach. $J$ Oncol Pract. 2016;12:1029-1038. doi:10.1200/JOP.2016.013664

10. Rotenstein LS, Zhang Y, Jacobson JO. Chronic comorbidity among patients with cancer: an impetus for oncology and primary care collaboration. JAMA Oncol. 2019;5:1099-1100. doi:10.1001/ jamaoncol.2019.1601

11. Schmidt M, Pedersen L, Sorensen HT. The Danish Civil Registration System as a tool in epidemiology. Eur $J$ Epidemiol. 2014;29:541-549. doi:10.1007/s10654-014-9930-3

12. da Cunha-bang C, Geisler CH, Enggaard L, et al. The Danish national chronic lymphocytic leukemia registry. Clin Epidemiol. 2016;8:561-565. doi:10.2147/CLEP.S99486

13. Gjerstorff ML. The Danish Cancer Registry. Scand J Public Health. 2011;39:42-45. doi:10.1177/1403494810393562

14. Pedersen CB. The Danish civil registration system. Scand J Public Health. 2011;39:22-25. doi:10.1177/1403494810387965

15. Schmidt M, Schmidt SA, Sandegaard JL, Ehrenstein V, Pedersen L, Sorensen HT. The Danish National Patient Registry: a review of content, data quality, and research potential. Clin Epidemiol. 2015;7:449-490. doi:10.2147/CLEP.S91125

16. Kildemoes HW, Sorensen HT, Hallas J. The Danish National Prescription Registry. Scand J Public Health. 2011;39:38-41. doi: $10.1177 / 1403494810394717$

17. Charlson ME, Pompei P, Ales KL, MacKenzie CR. A new method of classifying prognostic comorbidity in longitudinal studies: development and validation. J Chronic Dis. 1987;40:373-383. doi:10.1016/ 0021-9681(87)90171-8

18. Quan H, Li B, Couris CM, et al. Updating and validating the Charlson comorbidity index and score for risk adjustment in hospital discharge abstracts using data from 6 countries. Am J Epidemiol. 2011;173:676-682. doi:10.1093/aje/kwq433

19. Christensen KG, Fenger-Gron M, Flarup KR, Vedsted P. Use of general practice, diagnostic investigations and hospital services before and after cancer diagnosis - a population-based nationwide registry study of 127,000 incident adult cancer patients. BMC Health Serv Res. 2012;12:224. doi:10.1186/1472-6963-12-224

20. Michael Asger A, Casper Tabassum E, Christian B, et al. Incidence and predictors of infection among patients prior to treatment of chronic lymphocytic leukemia: a Danish nationwide cohort study. Haematologica. 2018;103:e300-e303. doi:10.3324/haematol.2017.182006

21. Agius R, Brieghel C, Andersen MA, et al. Machine learning can identify newly diagnosed patients with CLL at high risk of infection. Nat Commun. 2020;11:363. doi:10.1038/s41467-019-14225-8

22. Rotbain EC, Frederiksen H, Hjalgrim H, et al. IGHV mutational status and outcome for patients with chronic lymphocytic leukemia upon treatment: a Danish nationwide population-based study. Haematologica. 2020;105:1621-1629. doi:10.3324/haematol.2019.220194

23. Soo W-K, King M, Pope A, Parente P, Darzins P, Davis ID. Integrated geriatric assessment and treatment (INTEGERATE) in older people with cancer planned for systemic anticancer therapy. $J$ Clin Oncol. 2020;38:12011. doi:10.1200/JCO.2020.38.15_suppl.12011

24. Arts LPJ, Oerlemans S, Tick L, Koster A, Roerdink HTJ, van de Pollfranse LV. More frequent use of health care services among distressed compared with nondistressed survivors of lymphoma and chronic lymphocytic leukemia: results from the population-based PROFILES registry. Cancer. 2018;124:3016-3024. doi:10.1002/cncr.31410

25. Pfeil AM, Imfeld P, Pettengell R, et al. Trends in incidence and medical resource utilisation in patients with chronic lymphocytic leukaemia: insights from the UK Clinical Practice Research Datalink (CPRD). Ann Hematol. 2015;94:421-429. doi:10.1007/ s00277-014-2217-7 
26. Trabjerg TB, Jensen LH, Sondergaard J, Sisler JJ, Hansen DG. Improving continuity by bringing the cancer patient, general practitioner and oncologist together in a shared video-based consultation protocol for a randomised controlled trial. BMC Fam Pract. 2019;20:86. doi:10.1186/s12875-019-0978-8

27. Chen RC, Hoffman KE, Sher DJ, et al. Development of a standard survivorship care plan template for radiation oncologists. Pract Radiat Oncol. 2016;6:57-65. doi:10.1016/j.prro.2015.10.001

28. Cashman J, Wright J, Ring A. The treatment of co-morbidities in older patients with metastatic cancer. Support Care Cancer. 2010;18:651-655. doi:10.1007/s00520-010-0813-1

29. Puts MT, Costa-Lima B, Monette J, et al. Medication problems in older, newly diagnosed cancer patients in Canada: how common are they? A prospective pilot study. Drugs Aging. 2009;26:519-536. doi:10.2165/00002512-200926060-00008

30. Lu-Yao G, Nightingale G, Nikita N, et al. Relationship between polypharmacy and inpatient hospitalization among older adults with cancer treated with intravenous chemotherapy. J Geriatr Oncol. 2020;11:579-585. doi:10.1016/j.jgo.2020.03.001

31. Jørgensen TL, Herrstedt J. The influence of polypharmacy, potentially inappropriate medications, and drug interactions on treatment completion and prognosis in older patients with ovarian cancer. J Geriatr Oncol. 2020;11:593-602. doi:10.1016/j. jgo.2019.12.005

32. Goh I, Lai O, Chew L. Prevalence and Risk of Polypharmacy Among Elderly Cancer Patients Receiving Chemotherapy in Ambulatory Oncology Setting. Curr Oncol Rep. 2018;20:38. doi:10.1007/ s11912-018-0686-x

33. Lees J, Chan A. Polypharmacy in elderly patients with cancer: clinical implications and management. Lancet Oncol. 2011;12:1249-1257. doi:10.1016/S1470-2045(11)70040-7

34. Gaede P, Vedel P, Larsen N, Jensen GV, Parving HH, Pedersen O. Multifactorial intervention and cardiovascular disease in patients with type 2 diabetes. $N$ Engl J Med. 2003;348:383-393. doi:10.1056/ NEJMoa021778

35. Hershey DS, Tipton J, Given B, Davis E. Perceived impact of cancer treatment on diabetes self-management. Diabetes Educ. 2012;38:779-790. doi:10.1177/0145721712458835

36. Weiser MA, Cabanillas ME, Konopleva M, et al. Relation between the duration of remission and hyperglycemia during induction chemotherapy for acute lymphocytic leukemia with a hyperfractionated cyclophosphamide, vincristine, doxorubicin, and dexamethasone/ methotrexate-cytarabine regimen. Cancer. 2004;100:1179-1185. doi:10.1002/cncr.20071

37. Irizarry L, Li QE, Duncan I, et al. Effects of cancer comorbidity on disease management: making the case for diabetes education (a report from the SOAR program). Popul Health Manag. 2013;16:53-57. doi:10.1089/pop.2012.0028
38. Gallagher EJ, LeRoith D. THE INTERPLAY BETWEEN ENDOCRINOLOGY AND ONCOLOGY: THE NEW ROLE OF THE ENDOCRINOLOGIST. Endocr Pract. 2017;23:1167-1168. doi:10.4158/EP171997.ED

39. Aarup K, Rotbain EC, Enggaard L, et al. Real-world outcomes for 205 patients with chronic lymphocytic leukemia treated with ibrutinib. Eur J Haematol. 2020;105:646-654. doi:10.1111/ ejh.13499

40. Munir T, Brown JR, O'Brien S, et al. Final analysis from RESONATE: up to six years of follow-up on ibrutinib in patients with previously treated chronic lymphocytic leukemia or small lymphocytic lymphoma. Am J Hematol. 2019;94:1353-1363. doi:10.1002/ajh.25638

41. Dickerson T, Wiczer T, Waller A, et al. Hypertension and incident cardiovascular events following ibrutinib initiation. Blood. 2019;134:1919-1928. doi:10.1182/blood.2019000840

42. Burger JA, Barr PM, Robak T, et al. Long-term efficacy and safety of first-line ibrutinib treatment for patients with CLL/SLL: 5 years of follow-up from the Phase 3 RESONATE-2 study. Leukemia. 2020;34:787-798. doi:10.1038/s41375-019-0602-x

43. Earle CC, Neville BA. Under use of necessary care among cancer survivors. Cancer. 2004;101:1712-1719. doi:10.1002/cncr.20560

44. Snyder CF, Frick KD, Kantsiper ME, et al. Prevention, screening, and surveillance care for breast cancer survivors compared with controls: changes from 1998 to 2002. J Clin Oncol. 2009;27:1054-1061. doi: 10.1200/JCO.2008.18.0950

45. Earle CC, Burstein HJ, Winer EP, Weeks JC. Quality of non-breast cancer health maintenance among elderly breast cancer survivors. J Clin Oncol. 2003;21:1447-1451. doi:10.1200/ JCO.2003.03.060

46. Mato AR, Roeker LE, Allan JN, et al. Outcomes of front-line ibrutinib treated CLL patients excluded from landmark clinical trial. Am J Hematol. 2018;93:1394-1401. doi:10.1002/ajh.25261

47. Hallek M. Chronic lymphocytic leukemia: 2020 update on diagnosis, risk stratification and treatment. Am J Hematol. 2019;94:1266-1287. doi:10.1002/ajh.25595

48. Schiattone L, Ghia P, Scarfò L. The evolving treatment landscape of chronic lymphocytic leukemia. Curr Opin Oncol. 2019;31:568-573. doi:10.1097/CCO.0000000000000585

49. Herishanu Y, Shaulov A, Fineman R, et al. Frontline treatment with the combination obinutuzumab \pm chlorambucil for chronic lymphocytic leukemia outside clinical trials: results of a multinational, multicenter study by ERIC and the Israeli CLL study group. Am J Hematol. 2020;95:604-611. doi:10.1002/ajh.25766
Clinical Epidemiology

\section{Publish your work in this journal}

Clinical Epidemiology is an international, peer-reviewed, open access, online journal focusing on disease and drug epidemiology, identification of risk factors and screening procedures to develop optimal preventative initiatives and programs. Specific topics include: diagnosis, prognosis, treatment, screening, prevention, risk factor modification,

Submit your manuscript here: https://www.dovepress.com/clinical-epidemiology-journal systematic reviews, risk \& safety of medical interventions, epidemiology \& biostatistical methods, and evaluation of guidelines, translational medicine, health policies \& economic evaluations. The manuscript management system is completely online and includes a very quick and fair peer-review system, which is all easy to use. 